\title{
A Semiotic Multi-Agent System for Intelligent Building Control
}

\author{
Darren Booy \\ The University of Reading \\ Informatics Research \\ Centre, RG6 6BX \\ d.a.booy@rdg.ac.uk
}

\author{
Kecheng Liu \\ University of Reading \\ Informatics Research \\ Centre, RG6 6BX \\ k.liu@rdg.ac.uk
}

\author{
Bing Qiao \\ University of Reading \\ School of Systems \\ Engineering, RG6 6BX \\ b.qiao@rdg.ac.uk
}

\author{
Chris Guy \\ University of Reading \\ School of Systems \\ Engineering, RG6 6BX \\ c.g.guy@rdg.ac.uk
}

\begin{abstract}
Intelligent agents have often been used within intelligent buildings for autonomous actuation of heating, ventilation and air conditioning systems (HVAC) within intelligent buildings. Ubiquitous wireless sensors send environmental data such as temperature, which is then calculated by the agents in compliance to building policies and stakeholder requirements.

In this paper, we describe an organisational semiotic approach to designing intelligent agents and a proof of concept in MASBO (Multi-Agent System for Building cOntrol); a multi-agent system designed to provide coordinated management on Building control HVAC systems within an intelligent building. Overall, the system will provide automated assessment of the environment in realtime using readily deployable wireless sensors to personalize occupants' micro-environment and thus achieve optimal wellbeing and productivity.
\end{abstract}

\section{KEYWORDS}

Multi-agent systems, intelligent buildings, personalisation, semiotics, affordance, wireless sensor network. collaborative negotiation, actuators, pervasive space, ambient intelligence.

\section{INTRODUCTION}

The productivity of occupants within any working environment can be directly related to their environmental conditions. Intelligent sustainable healthy buildings improve business value because they respect environmental and social needs and occupants' well-being, which improves work productivity and human performance [1]

Occupant productivity can be improved through personalisation of their working space which can be carried out by monitoring the level of well-being using intelligent

Permission to make digital or hard copies of all or part of this work for personal or classroom use is granted without fee provided that copies are not made or distributed for profit or commercial advantage and that copies bear this notice and the full citation on the first page. To copy otherwise, to republish, to post on servers or to redistribute to lists, requires prior specific permission and/or a fee. AMBI-SYS 2008, February 11-13, Quebec, Canada

Copyright (C) 2008 ICST 978-963-9799-16-5

DOI 10.4108/ICST.AMBISYS2008.2881 autonomous agents which use an ambient wireless sensor network in real time and can actuate the environment to suit the occupant's requirements. The empowerment of the occupant through self evaluation focuses on improving productivity of the occupant autonomously whilst maximising energy efficiency. The multi-agent system for building control (MASBO) facilitates autonomous cooperation between the occupant and the intelligent building through actuation of the building's HVAC system, in accordance to occupant requirements organised within predefined occupant zones/areas specified at design stage of the building. These zones are specified between stakeholders at the preoccupancy stage, in order to create a more manageable space to control. This captured zone data is also the centre of negotiation between stakeholders through a Collaborative design environment (CDE) that allows stakeholders to evaluate their environment and continuously negotiate them for each predefined areas or zones of a building with other stakeholders. The range of these requirements also complies with any boundaries of actuation the building policies enforce. In our approach towards developing the agent architecture for MASBO, we have used the EDA model. The EDA model (Epistemic, Deontic and Axiological) is based upon social attitudes and norms; it also incorporates other fundamental semiotic principles such as affordance. The agent system itself consists of multiple agents working congruently but undertaking different roles which are detailed later in the text.

In this paper we describe an organisational semiotic approach to designing intelligent agents based on semiotic fundamentals such as social norms. These Intelligent agents are able to monitor, negotiate HVAC settings and adapt building control systems for occupants within an intelligent pervasive space that best suits their optimum productive environment and react autonomously.

This paper sources research from two ongoing projects; CMIPS (Coordinated Management of Intelligent Pervasive Spaces) and IDCOP (Innovation in Design, Construction \& Operation of Buildings for People). 


\section{REVIEW OF PREVIOUS WORK}

Research work conducted by Davidsson and Boman [2] provides a useful inspiration on using a multi-agent system to control an Intelligent Building. It is part of the ISES (Information/Society/Energy/System) project that aims to achieve both energy saving and customer satisfaction via value added services. Energy saving is realized by automatic control of lighting and heating devices according to the presence of occupants, while customer satisfaction is realized by adapting light intensity and room temperature according to occupants' personal preferences. In $[2,3]$ a soft computing architecture is discussed, based on a combination of DAI (distributed artificial intelligence), fuzzy-genetic driven embedded-agents and IP internet technology for intelligent buildings.

This research also presents another feature in some cases preferable for intelligent building environment: user interaction and feedback to the MAS. However its use of embedded agents makes it difficult to take advantage of sophisticated agent platforms and as claimed by the researchers, places severe constraints on the possible AI solutions.

Further research following [2] is the iDorm project [4], where an intelligent dormitory is developed as a test bed for a multiuse ubiquitous computing environment. One of improvements of iDorm over [4] is the introduction of iDorm gateway server that overcomes many of the practical problems of mixing networks. However, iDorm is still based on embedded agents, which despite demonstrating learning and autonomous behaviours, are running on nodes with very limited capacity.

The flexibility of the EDA Model has enabled it to be applied to multiple disciplines. The most common usage however has been within knowledge representation for intelligent agents targeted to towards mimicking human rationality in research [5], [6], [7].

There are apparent similarities between the EDA Model and the more well known BDI model. The BDI (beliefdesire-intention) model is well understood as agent architecture to support goal oriented behaviour in intelligent agents. It provides a folk psychological way by simulating the human way of making decisions [8]. The BDI and the EDA model depict similar components such as a belief (can be related to perceptual), desire (objective \& method) and Intention (evaluation \& execution). However, instead of manipulating the notions of belief, intention, desire, commitment, goal and obligation, the only primitives that we need are belief and generalised goal. [6].

\section{MASBO OVERVIEW}

In the MASBO example the multi agent system consists of four main roles the Monitor \& Control agent, Central Agent, Local Agent and Personal Agent in which they interact with each other through affording occupation that is required for successful occupant recommendation or actuation.

This multi-agent system acts as an add-on to existing BMS (Building Management System) and requires inputs from agreed policies and assessment systems. The multi-agent system aims to dynamically configure building facilities to meet the requirements for building energy efficiency and personalised work environment, Figure 1 shows the functional architecture of MASBO.

The central agent is the interface of the multi-agent system to BMS and the number of central agent depends on the decomposition of the functionality required. While there will be only one local agent for each zone, every environmental parameter for a zone will require a dedicated monitor \& control agent, preferably running on a mobile device, the personal agent will be equipped for every occupant in a building.

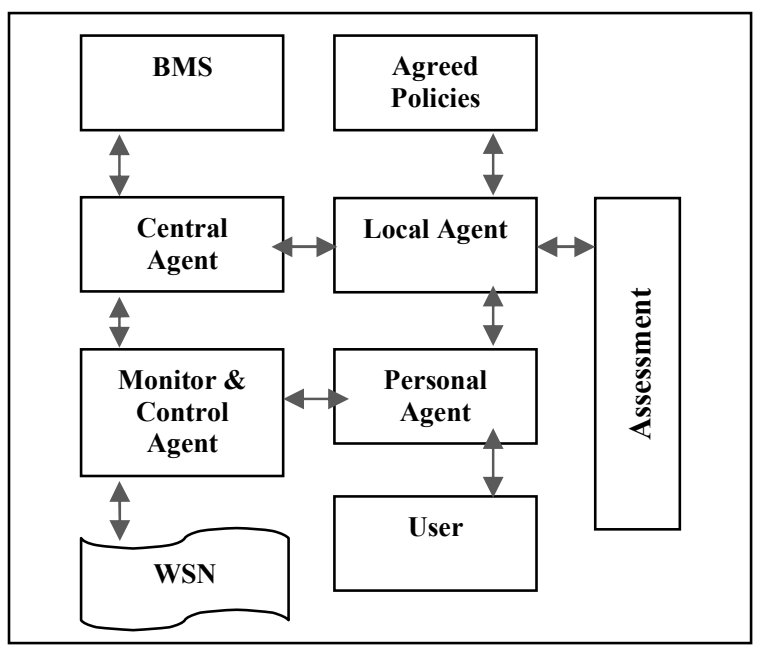

Figure 1: The MASBO Architecture

Each agent within MASBO has been developed in accordance to the EDA Model Architecture depicting and communicating rationally between Deontic and Perceptive data sent by other agents. The fundamental role of each element of the EDA Model applied to each agent functions the same throughout but differing in affordances available to it such as the WSN to the Monitor control agent or the agreed policies to the local agent.

\section{COLLABORATIVE DESIGN ENVIRONMENT}

The purpose of the Collaborative Design Environment (CDE) is to empower major stakeholder groups e.g. main occupant, facilities manager, business manager, owner and visiting occupants by allowing them to specify personal environmental requirements for specific occupant zones of a building, with the overall objective through successful negotiation with other stakeholders to actuate a HVAC system intelligently. The conventions which surround 
the requirements specification for the $\mathrm{CDE}$ are mainly related to the current building's facilities management policies within whatever domain the CDE would be functioning. This convention would be regulated autonomously by the agent system, supervising stakeholder requirements specification deontically through the EDA agent architecture and offering notification to the user if any specification contradicts any current existing building policies. Overall the Facilities Management Supervisor would be in main control of the constraints related to these conventions. The CDE would also convene to minimum usability guidelines to increase the possibility of success.

The proposed functional requirements for the CDE are as follows:

- To depict the sensor data clearly and accurately.

- To allow stakeholders to specify their environmental requirements within an intelligent building.

- To allow stakeholders to negotiate these requirements with other stakeholders.

- To allow stakeholders to select zones within an intelligent building for potential requirement specification.

Successful Stakeholder negotiation of each zone is dependant on clear and accurate depiction of the HVAC settings. The final value of the Stakeholder negotiation is dependant on other stakeholders' specification and existing building policies.

Working alongside the CDE is a multi agent system (MASBO) in which their role is to provide feedback to the user based on their previous successful settings. Its function is also to stabilise the environment from these existing settings of the HVAC system when stakeholder negotiation is not currently taking place. The MASBO system implements the EDA model as an agent architecture which is based upon social norms and attitudes.

An overall architecture of the intelligent system of both the CDE and MASBO working congruently can be viewed in Figure 2. The user(s) initially specify a zone to be actuated understood from the wireless sensor data by displaying clearly within the CDE and also sending it to MASBO (Monitor \& Control agent). From accurate depiction of this real time data, stakeholders are then able to negotiate HVAC settings for this zone. During this process the Local Agent from MASBO also makes recommendations to the stakeholders what actually previous HVAC settings had been successful in accordance with the agreed policies set by the BMS. Deductions from MASBO also use this data from the WSN in order to stabilize the zone itself. Upon successful stakeholder negotiation which is completed via averaging specification also in accordance with the agreed policies, actuation eventually takes place.
This process is repeated and the stakeholders are continuously and unobtrusively updated by MASBO with feedback of previous productive environments. Stakeholders can also choose to negotiate these recommendations.

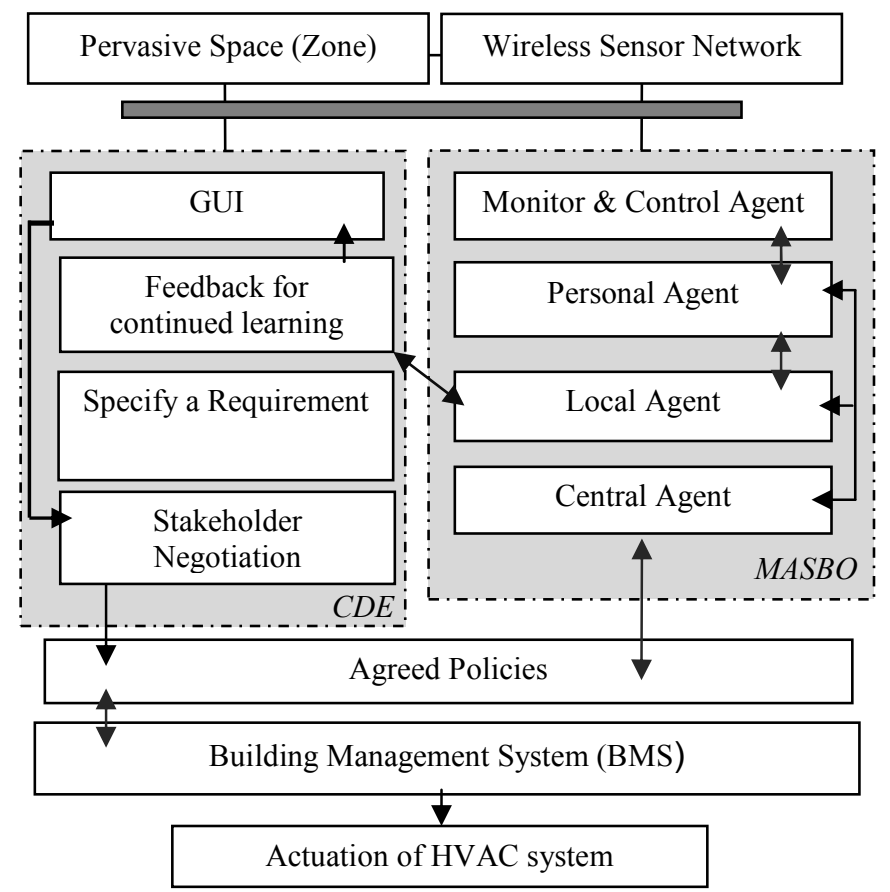

Figure 2: Example of EDA to Intelligent Building Control

\section{SEMIOTICS FOR INTELLIGENT AGENTS}

Semiotics is the study of human use of signs and symbols, and also humans' reaction to the interpretation of signs employed in communication and coordination. A general definition of semiotics can be depicted as 'a general philosophical theory of signs and symbols that deals especially with their function in both artificially constructed and natural languages and comprises syntactics, semantics, and pragmatics.' [9]. This definition can be related to most scenarios in the $21^{\text {st }}$ century in which we are ubiquitously surrounded by signs and intuitive complex signs - buildings are such examples of complex signs. Organisational semiotics [10],[11], a subdivision of semiotics, which focuses on the analysis of organised activities in business settings, coheres with our research more resourcefully. Two notions (the latter is rooted in organizational semiotics) have to be introduced in order to appreciate the buildings as a complex sign:

- A Sign is something which stands to somebody for something else in some respect or capacity

- A Norm is generalised disposition to the world shared by members of the community. [10] 
From these two fundamentals a simple example to elucidate this would be of mercury rising and falling on a thermometer. The purpose of the mercury is in itself a sign to indicate a position on a scale of scientific convention (norm). Without this norm in place, the purpose of the mercury is obsolete or the function of the artefact changes depending on the social construct of the user. The successful outcome from the merge of these two principles and a physical input in this case (temperature) results in a truthful temperature reading. The same principle can be applied to how intelligent agents operate within our agent architecture. Intelligent agents within the intelligent buildings initially perceive signs (environmental data) from the wireless sensor network (WSN) and then compares it to previous logical deductions whilst also comparing which signs to perceive, which actions to execute and also the obligation the agent has to the current policy of the building (norm). This deduction is then combined and evaluated by the set of available plans, either explicitly or implicitly, that the agent may choose to execute.

A fundamental aspect of semiotics is the concept of affordance. Since its introduction to the world of perceptual theory, affordance is becoming more pervasive within the computational field and has been applied to areas such as artificial intelligence, robotics and user interfaces [12]. Systems which require a significant human interaction have also adopted the notion of agent affordance. This concept enables the developer to account the outcome of agent reasoning due to the actions available to it. There has been substantial research dedicated to the field of agent affordance for use within artificial intelligence to take the raw data, work out the context, plan how to carry out the operation and assign tasks to subordinate agents [9]. Our research concentrates on the architecture for the representation of the agents denoting the same affordances as the occupant but denoting different deductions (Human deduction \& EDA model for intelligent agents).

\section{THE EDA AGENT ARCHITECTURE MODEL}

These elements of semiotics can be further expanded into four basic component modules; Perceptual, Axiological, Epistemic and Deontic originally based around a set of social norms. The EDA model is based upon the basic principle upon that 'information is a complex concept, and requires different viewpoints to be completely analyzed' [10]. Using this view point the framework conceptualises information interpretation from various angles and is mainly derived from pragmatic analysis and perlocutionary effects between agent communicative relationships. The EDA model exemplifies the agent informational states and simultaneously classifies the relationship between them. These components have been modelled to form the agent architecture used for each agent within MASBO described in further detail later in this paper. The perceptual interface is the initial element which perceives the set of data that has been received from the wireless sensor network or other agents. The Epistemic element of the agent architecture is the existing knowledge and beliefs the agent attained from previous user requirements and data supplied from the WSN.

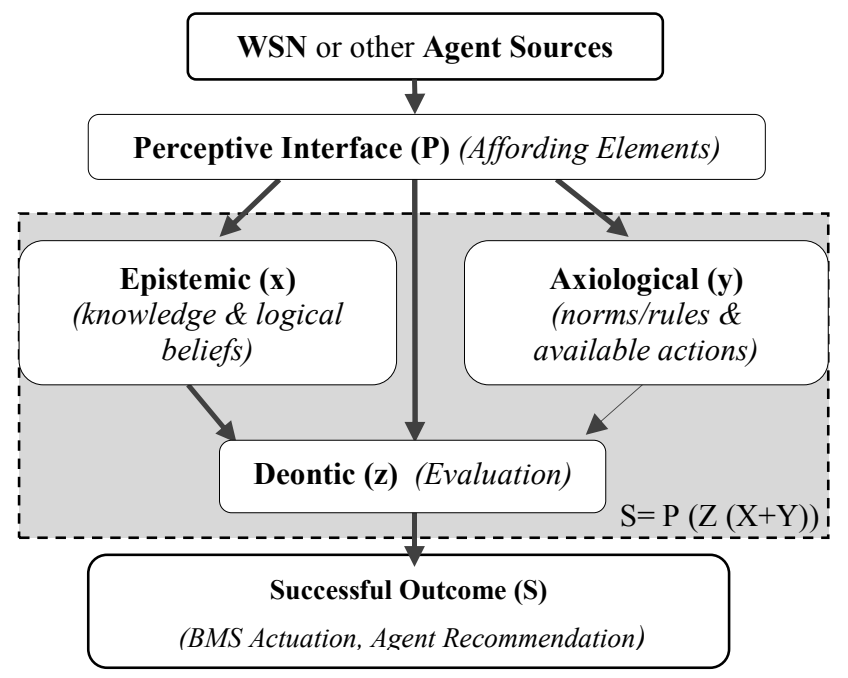

Figure 3: The EDA Agent Model

Axiological principles of the diagram depict which signs the agent or agents should perceive and what actions are currently available. This is essentially important as the agent may be bound to current building policies set by the facilities management department. These constraints may be part of a bigger plan according to building consumption or to enhance occupant productivity.

Deontic focuses on the obligation of what actions the agent is able to perform based on the combination of the perceptual interface, epistemic logical beliefs and axiological norms. The outcome of a deontic evaluation may result in the building control system actuating a HVAC system such as a temperature setting according to a stakeholder requirement or possibly an agent recommendation to the user dependant on the initial relative goal which may vary depending on which agent the EDA model has been applied to.

\section{AFFORDANCE APPLIED TO INTELLIGENT AGENTS}

The agents used within the system are rational agents in which decisions are based upon past experiences (record of user preference) and information from the environment (sensor network) to increase the chances of successful autonomy for the occupant. A fundamental constituent of Semiotics is a term called 'affordance' which can be defined as a characteristic of an object, esp. relating to its potential utility, which can be inferred from visual or other 
perceptual signals; (more widely) a quality or utility which is readily apparent or available [13]. We use the term affordance to describe a potential for action, the perceived capacity of an object to enable the assertive will of the actor. The term was coined by psychologist James Gibson [14] to depict possibilities of action posed by artefacts in the real world.

When applied to agents, affordances can be defined as the capabilities that an agent is able to respond within its environment. The intelligent agents' role within our system is to mimic an occupant's requirements that belong to a specified zone. Therefore the agent's affordance must be directly related to how the occupant perceives or interplays with their environment through its affordances. The occupant and the agent's affordance would vary depending on their privileges within the building, e.g. a security guard is able to unlock any door if required due to no limitations of access. The environment in which the agent is a part of, affords capability if the agent meets the requirements of two principles:

1. It has the capacity to recognize that it is in such a relation between itself and its environment and it

2. It has the ability to act to bring about that capability. [15]

The affordances for each agent vary depending on their role within MASBO for example the Monitor \& Control agent affords data from the WSN which acts as an intermediate channel between the stakeholders specified zone's environmental state and other agents within MASBO which are also affordances to this agent. Combining the concept of affordance applied to intelligent agents and the organisational semiotic approach of the EDA model both within MASBO results in a powerful analysis of intelligent agent design. A breakdown of each agent within MASBO is detailed below.

\section{- 7.1 Monitor \& Control Agent}

This agent enforces the operation request given by the user, reads and processes sensor data, and achieves an environmental state according to decisions made by the Local Agent. [18] Affordances available: WSN, Personal Agent, Local Agent, Central Agent.

Perceptive Element of Agent: Monitors data from the WSN and other agents (central, local, personal) in the form of user behaviour, operation request and agent requests etc.)

Epistemic Element of Agent: Retrieves previous data from WSN and local \& personal agents (eg. previous user behaviour, previous successful HVAC configuration etc.) then sends to deontic element for comparison.

Axiological Element of Agent: Checks values from current building policies and sets available actions for deontic comparison.
Deontic Element of Agent: Evaluates from previous elements and processes data from WSN to be sent to corresponding agents or external systems.

\section{- $\quad 7.2$ Personal Agent}

Manages user (occupant) profile, observes the work environment, records user's behaviour, forwards operation requests, learn preferences and presents feedback from other agents to the occupant.[18]

Affordances available: Monitor Control Agent, Local Agent, User.

Perceptive Element of Agent: Monitors data from other agents (local, M\&C) e.g. deductions, recommendations and operation requests from user, also observes environment though Monitor \& Control agent.

Epistemic Element of Agent: Retrieves previous data from occupant profile including past preferences and user operation requests and then sends to deontic element for comparison.

Axiological Element of Agent: Checks values from building policies currently in place and past user specification then sends available actions for deontic comparison.

Deontic Element of Agent: Evaluates from previous elements and recommends an action to corresponding agents in the form of feedback to the collaborative design environment for the user to assess.

\section{- $\quad$ 7.3 Local Agent}

This agent plays a central role in MASBO. It acts as a mediator, policy enforcer and information provider. It reconciles contending preferences from different users, enforces policies that constrain the environment parameters, provides structural information for their respective zones, and responds to environmental state change. [18]

Affordances Available: Personal Agent, Monitor Control agent, Central Agent, Policy Management

Perceptive Element of Agent: Monitors data from other agents (central, personal, M\&C) in the form of user operation requests (from personal agent), agent system configurations (from central agent) and WSN deductions (from M\&C agent). It also affords any environmental state change that other agents have actuated.

Epistemic Element of Agent: Retrieves past recommendations from contending preferences between user operation requests, environmental parameters that had been previously used and also related structural information (zones) and then sends to deontic element for comparison.

Axiological Element of Agent

Enforces current building policies in place and user operation limitations then sends available actions for potential actuation and deontic comparison.

Deontic Element of Agent

Evaluates from previous elements and reconciles any conflict of user preferences alongside any building policy management then recommends an action to corresponding agents and to mediate stakeholder negotiation. 


\section{- $\quad$ 7.4 Central Agent:}

This agent has two major functions: decision aggregation and interface to internal/external services required by other agents. The typical services provided by central agent include agent system configuration and interface to BMS. [18]

\section{Affordances Available: BMS, Local Agent, Monitor \& Control Agent}

Perceptive Element of Agent: Monitors data from other agents (local, M\&C) in the form of previous agent recommendations (from local agent), and current environmental data from WSN (M\&C agent).

Epistemic Element of Agent: Retrieves ontological data from internal/external services of the BMS and past data from agent configuration.

Axiological Element of Agent: Checks data from other agent recommendations then sends available actions for deontic comparison.

Deontic Element of Agent: Aggregates decisions made by other agents received from previous elements and interfaces with internal/external services required by other agents

\section{CONCLUSION AND FUTURE WORK}

Many efforts have been made on using multi-agent system for intelligent building control. However, while overall the previous work has addressed most of the important features for the MAS based intelligent building control, we claim that no research has been done to consider all the following requirements:

- Human readable and accurate knowledge representation

- Energy efficiency and occupants' comfort

- Non intrusive preferences learning

- Personalized control and feedback

- State of the art implementation techniques for developing the agent platform

We believe above requirements are essential to a successful intelligent building environment and a complete solution should tackle all of them.

Further development regarding the MASBO implementation includes:

- Animated simulation to be implemented in the Web portal.

- Mobile application to be further developed and tested on a smart phone.

- Integrator. At this stage, only a subset of query messages is implemented. The full set of query messages will be implemented for second integration plan.

Finally the prototype development of a collaborative design environment (CDE) to be used for capturing, modelling and analysing stakeholders requirements which plays an essential deliverable of our research and is the forefront application of the interaction between the occupant and the intelligent agent rich environment using the CMIPs architecture as a backbone to the system.

Both MASBO and CDE are primarily focused on the occupant's personal profile adapting and updating autonomously to stakeholders' optimum working environment though personalisation and negotiation. Tables 1 and 2 [19], [20] provide an example of post occupancy evaluation and provide an indication on the representation of data within the domain that MAS and stakeholder may interplay with.

\begin{tabular}{|l|l|}
\hline Sense & Typical parameters for an office \\
\hline $\begin{array}{l}\text { Temperature } \\
\text { (ambient) }\end{array}$ & $\begin{array}{l}22-24^{\circ} \mathrm{C} \text { Summer, } \\
21-23^{\circ} \mathrm{C} \text { Winter }\end{array}$ \\
\hline $\begin{array}{l}\text { Light (desk } \\
\text { surface } \\
\text { height) }\end{array}$ & $300-500 \mathrm{Lux}$ \\
\hline Noise & $35 \mathrm{NR}$ (or around $45 \mathrm{~dB}$ ) \\
\hline Air flow & $1.25-2$ changes per hour \\
\hline Humidity & $40-60 \%$ \\
\hline $\begin{array}{l}\text { Indoor Air } \\
\text { Quality }\end{array}$ & $\begin{array}{l}8 \text { litres per sec per person (assumes } 1 \\
\text { person per 10m2 office) } 700-1000 \mathrm{ppm} \\
\text { CO2 (parts per million) }\end{array}$ \\
\hline
\end{tabular}

Table 1. Quantitative parameters

Table 1 lists the most common environmental parameters within a building domain that can be measured in a quantitative manner. These parameters reflect the recommended range of BMS actuation developed by Arup's Integrated Workplace Performance research [20]. There has also been further analysis which may effect the occupant's perception of their environment such as job stress or overcrowding resulting in an increase in the occupants' thermo-response which also varies across gender, medical history and age. [21]

\begin{tabular}{|l|l|}
\hline Sense 7 point scale & \\
\hline Temperature & Hot - cold \\
\hline Light & Dark - bright \\
\hline Noise & Noisy - quiet \\
\hline Humidity & $40-60 \%$ \\
\hline Air Quality & Good - bad \\
\hline Overall satisfaction & Good - bad \\
\hline
\end{tabular}

Table 2. Qualitative parameters

Table 2 depicts potential qualitative records that can be used by occupants in self evaluation of their environment in real time. These inputs can be used by the personal agents to recommend a previous successful environment settings rather than the quantitative approach outlined in Table 1.

The requirement of user feedback or interactions in intelligent building environment is controversial. Some researchers claim that ambient intelligence should not be 
intrusive, i.e., no special devices used and no imposing rules on occupants' behaviour. In [16], a multi-agent system is discussed for intelligent building control. Occupants tend to prefer direct control over their environment via physical interaction e.g. opening a window. The representation of the agent autonomy via the CDE is critical to deter this social attitude. The provision of autonomous agents has also received negative emotional effects. It is clear, that in any human-agent interaction the agent needs to take account of the human's likely feelings towards any intervention. Simply giving information that "should" be helpful, in terms of task efficiency, speed, etc., is not sufficient [16]. In contrast to the approach in [7], the MAS is equipped with an unsupervised online real-time learning algorithm that constructs a fuzzy rule-base, derived from very sparse data in a non-stationary environment. All feedback is acquired by means of observing occupants' behaviours without intruding on them, fully cooperative at all times. The agent is be available when the occupant requires and ambient but productive to suit the user's specification when the user is focusing on a different task which ultimately is aiding the user in adapting the subsystems to create an optimum personalised environment for productivity. Agent activity would be made apparent at any time if the user wishes to evaluate its performance.

Semiotics would play a critical part in empowering occupants with a resource of evaluation of their environment and also unobtrusively informing them of their current conditions whilst adapting their environment to suit their personalised optimum conditions for productivity. Since applications in intelligent environments need to reason about context and have constraints based upon changing policies, we need to develop methods of representing individual, organizational and societal norms. [17]

\section{ACKNOWLEDGMENTS}

This work was partly supported by EPSRC (Grant GR/T04878/01); Innovation, Design and Operation of Buildings for People. Dr Penny Noy had contributed to the research during the first year of the project, The University of Greenwich and The University of Southampton are other participating institute on this project. The also benefited from CMIPS, a collaborative project sponsored by DTI (British Department of Trade and Industry, Grant number: TP/3/PIT/6/1/16218), participated by Thales Research \& Technology, Arup and University of Reading within the School of Systems \& Engineering.

\section{REFERENCES}

1. Bing Qiao, K.L., Chris Guy. Multi-agent Building Control in a Shared Environment. in IAT 2006. 2006. Beijing, China: IEEE.

2. Boman, P.D.a.M., "Distributed Monitoring and Control of Office Buildings by Embedded Agents.” Information Sciences, 2005. 171: p. 293-307.
3. Filipe, J. A Normative and Intentional Agent Model for Organisation Modelling. in Engineering Societies in the Agents World III: Third International Workshop. 2002. Madrid, Spain.

4. H. Hagras, V.C., M. Colley, G. Clarke, A. Pounds-Cornish and H. Duman, Creating an Ambient-Intelligence Environment Using Embedded Agents. $2004 . \quad$ (19): p. 12-20.

5. Huang, H.Z.a.S.Y. A General Framework for Parallel BDI Agents. in IAT 06. 2006. Beijiing, China.

6. Liu, K., Virtual, Distributed and Flexible Organisations: Studies in Organisational Semiotics. 2004, Kluwer Academic Publishers.

7. V V. Callaghan, G. Clarke, M. Colley and H. Hagras, "A SoftComputing DAI Architecture for Intelligent Buildings," Sof Computing Agents: New Trends for Designing Autonomous Systems, Springer-Verlag, 2001.Amant, R.S. Planning and user interface affordances. in International Conference on Intelligent User Interfaces. 1999. Redondo Beach, Los Angeles, CA.

8. Miriam Webster online dictionary, http://www.m-w.com/cgibin/dictionary?

9. Filipe J, L. K. (2000). The EDA Model: An Organizational Semiotics Perspective To Norm-Based Agent Design, Proceedings of the Agents. Workshop on Norms and Institutions in Multi-Agent Systems. Barcelona, Spain.

10. Liu K, (2004) Kluwer Academic Publisher Virtual, Distributed and Flexible Organizations: Studies in Organisational Semiotics, ISBN: 978-1402021619

11. Llu Frank, L.O.D., (2002). Mapping Cognitive Work Analysis (CWA) To An Intelligent Agents Software Architecture: Command Agents. in Proceedings of the Defence Human Factors Special Interest Group (DHFSIG) 2002. DSTO

12. OED (2007). The Oxford English Dictionary. (searched term 'affordance'.

13. Gibson, J.J., The theory of affordances. Perceiving, Acting and Knowing. , ed. n.R.S.J.B. (eds.). 1977, Hillsdale, NJ: Erlbaum.

14. Gibson, J.J., The theory of affordances. Perceiving, Acting and Knowing., ed. n.R.S.J.B. (eds.). 1977, Hillsdale, NJ: Erlbaum.

15. U. Rutishauser, J. Joller and R. Douglas, "Control and Learning of Ambience by an Intelligent Building," IEEETransactions on Systems, Man, and Cybernetics, Part A:Systems and Humans, 35, 121-132, 2005

16. P. Noy, K. L., D. Clements-Croome, B. Qiao (2006). Design Issues In Personalizing Intelligent Buildings. $2^{\text {nd }}$ International Conference on Intelligent Environments IE06, Athens, Greece.

17. Liu K, (2004) Kluwer Academic Publishers, Virtual, Distributed and Flexible Organizations: Studies in Organisational Semiotics, ISBN: 978- 1402021619

18. Co-ordinated Management of Intelligent Pervasive Spaces," published in the Proceedings of The 5th International Conference on Industrial Informatics, 23-27, July 2007, Vienna, Austria.

19. "Environmental design - CIBSE Guide A", The Chartered Institution of Building Service Engineers, London, 2006, ISBN-10:1-90328766-9

20. "An integrated framework to improve the workplace", Facilities Management, January 2006 ARUP.

21. "Intelligent Buildings And Indoor Air Quality" Prof Derek Clements-Croome, The University of Reading, http://www.extra.rdg.ac.uk/ib/Links\%20and\%20Downloads/Intellige nt\%20Buildings\%20and\%20Indoor\%20Air\%20Quality.pdf 